\title{
Perfil del docente en el enfoque basado en competencias
}

\section{Teacher's Profile based on Competences}

\author{
Margarita María Álvarez ${ }^{1}$ \\ Universidad Nacional de Santiago del Estero \\ Santiago del Estero, Argentina \\ alvarez@unse.edu.ar
}

Recibido 03 de noviembre de 2010 • Aceptado 09 de marzo de 2011

\begin{abstract}
Resumen. La educación basada en competencias contiene el potencial para convertirse en un plan eficaz tendiente a mejorar el aprendizaje del estudiantado y, de esta manera, prepararle para abordar el trabajo futuro. Pero este enfoque requiere de un largo proceso de reformas, lo que implica, necesariamente, la transformación de los actores educativos: docentes y estudiantes. Ya que grupo de docentes constituye uno de los pilares de la educación basada en competencias, en este artículo se presentan las necesarias características o perfiles de docentes universitarios para abordar este enfoque.
\end{abstract}

Palabras claves. Docente, enfoque basado en competencias, universidad, perfil.

Abstract. Competency-based education holds the potential to become an effective plan aimed at improving the learning of students and, thus preparing students to address future work. However, this approach requires a long process of reforms, which necessarily implies the transformation of the education participants: teachers and students. Since the group of teachers is a fundamental part of competency-based education, this study presents the profile or characteristics necessary in university teachers.

Keywords. Teacher, competency-based approach, university, profile.

\section{Introducción}

La sociedad actual está en un proceso de readaptación, desde un modelo industrial a una sociedad del conocimiento. El tratado del libre comercio, la globalización y la celeridad con la que se desarrolla la tecnología de la información y comunicación, marcaron el comienzo del siglo XXI $\mathrm{y}$, con ello, el cambio educativo que tiene como reto transformar y cambiar la manera de formar individuos, de tal suerte que se les proporcionen los elementos necesarios para vivir en una sociedad competitiva (Rodríguez, Aguilar, Jiménez y Pérez, 2001). Por lo tanto, se hace necesario “(...) un cambio urgente de actuación de las universidades, de los docentes y del resto de los interlocutores de

\footnotetext{
Ingeniera en Computación otorgado por la Universidad Católica de Santiago del Estero. Argentina. Máster en Ingeniería de Software otorgado por la Universidad Politécnica de Madrid, España. Especialista en Docencia Universitaria otorgado por la Universidad Nacional de Cuyo, Mendoza. Argentina. Es docente desde hace varios años en asignaturas de las carreras de Licenciatura en Sistema de Información y Profesorado en Informática de la Universidad Nacional de Santiago del Estero. Ha dirigido y asesorado varios trabajos de graduación. Ha participado en varios proyectos de investigación en la Universidad Nacional de Santiago del Estero y en dos de ellos como coordinadora de un subproyecto. Dispone de comunicaciones y publicaciones. Actualmente es integrante de la Comisión Asesora de la Escuela de Informática, y participa en la reestructuración de la Carrera de Licenciatura en Sistema de Información para otorgar títulos intermedios.
} 
la comunidad educativa" (Ortega, 2010, p. 306), que afronte la educación con nuevas concepciones y estrategias didácticas.

Para dar respuesta a estas necesidades, desde la década pasada, a partir de la Declaración de Bolonia (1999) y el Proyecto Tuning América Latina (2005) surgió la educación basada en competencias. El Proyecto Tuning Europeo integró a más de 175 universidades europeas y creó el Espacio Europeo de Educación Superior, cuyo objetivo principal fue generar un espacio para permitir acordar, templar y afinar las estructuras educativas en cuanto a las titulaciones, de manera que estas pudieran ser comprendidas, comparadas y reconocidas en el área común europea.

La educación basada en competencias contiene el potencial para convertirse en un plan eficaz tendiente a mejorar el aprendizaje del estudiantado y debe ser un reto que hay que aceptar e integrar en la cultura académica, ya que se tendría un vigoroso instrumento para diseñar currículos innovadores, fortalecer el aprendizaje y, con ello, acortar la distancia que se ha ido abriendo entre la educación universitaria y la práctica profesional (López y Farfán, 2005).

Pero este enfoque requiere un largo proceso de reformas -tanto educativas como institucionales-, lo que implica, necesariamente, la transformación de los actores educativos. Entre los que se debe mencionar destacan docentes y estudiantes. El primer grupo, en cuanto a su filosofía con respecto de la educación y a su manera tradicional de enseñar; y el estudiantado, en la responsabilidad de su propio aprendizaje.

La preocupación por cómo debe ser y actuar el profesor, y cuáles deben ser las características personales y profesionales que le configuran como profesional, han sido y son temas de investigación de autores como Galvis (2007); Ortega (2010); Pereda (s. f.) y Segura (2004). Por lo que, en el presente estudio se realiza una aproximación a algunas características o perfiles docentes universitarios necesarios para abordar el enfoque basado en competencias.

\section{Desarrollo}

\section{El enfoque basado en competencias}

Ante las demandas generadas en el contexto de globalización, surge la educación basada en competencias que, a fin de responder a las nuevas exigencias, propone una educación flexible, abierta y estrechamente relacionada con los sectores productivos. Esto ha originado que en muchos países, sobre todo en Europa, se implementen estrategias para mejorar la calidad educativa, tales como, transformaciones en los planes de estudio, cambios rotundos en el trabajo docente y, en general, en la calidad del servicio que ofrecen, todo ello orientado a la formación de profesionales de alta calidad (Martell, 2010).

(...) Las competencias constituyen la base fundamental para orientar el currículo, la docencia, el aprendizaje y la evaluación desde un marco de calidad, ya que brinda principios, indicadores y herramientas para hacerlo, más que cualquier otro enfoque educativo. (Tobón, 2006, p. 1)

Por su parte, Bogoya, citado por Martell (2010), explica que el desarrollo de competencias lleva al individuo, de una manera pertinente y oportuna, a la apropiación y aplicación del conocimiento para la resolución de problemas. Así, es posible considerar las competencias como "(...) procesos complejos de desempeño con idoneidad en un determinado contexto, con responsabilidad (...)” (Tobón, 2006, p. 5). 
La formación basada en competencias está en el centro de una serie de transformaciones en la educación, lo que requiere de profundos cambios, de nuevos perfiles tanto de estudiantes como de docentes. Pero, para poder abordar los perfiles que el docente universitario debería poseer en la educación basada en competencias, en este estudio se considera primordial conceptualizar al estudiante, con sus perfiles y aprendizajes.

\section{Breve conceptualización del estudiante y la estudiante}

La educación basada en el desempeño pasa de la enseñanza al aprendizaje. Pone énfasis en la actitud del estudiante y la estudiante, quienes dejan de ser pasivos y receptores para convertirse en activos, co-protagonistas y responsables de su propio aprendizaje. Individuos creativos, críticos, reflexivos, capaces de resolver problemas y con ansias de superación.

El aprendizaje ya no es sólo del texto o del discurso del docente, sino que se hace, fundamentalmente, del contexto, desde la propia cultura y desde la propia experiencia, un aprendizaje a lo largo de toda la vida. Se aprende, ya no en soledad, sino interactuando, colaborando, expresándose, relacionándose. En la formación basada en competencias, cada estudiante debe experimentar una variedad de enfoques y tener acceso a diferentes contextos de aprendizaje, sea cual sea su área de estudio.

\section{Perfil de la docente y del docente universitarios}

La educación basada en el desempeño, no sólo está centrada en el estudiantado, sino también en el rol docente. Compromete a este en la modificación de su práctica docente, su manera de diseñar las actividades y estrategias, su planeación no como un mero requisito administrativo, sino como un referente de cómo conducir al estudiantado en la consecución de los objetivos, propósitos y en el desarrollo de sus competencias y conocimientos, de forma tal que les sirvan para enfrentar y responder a determinados problemas presentes a lo largo su vida. “(...) Básicamente, nos compromete a modificar nuestra actitud hacia las estrategias que utilizamos para cómo conducir la enseñanza, esa estrategia que tenemos muy arraigada en nuestra práctica docente y que no se ha ido actualizando" (Zenteno, 2009, p. 4). Muchas de esas actitudes se deben a una resistencia al cambio, ya sea por haber sido educados con otros enfoques, por desconocimiento o por no estar de acuerdo con el enfoque basado en competencias.

Sin embargo, se considera que el papel del docente es el

(...) de un agente de cambio que entiende, promueve, orienta y da sentido al cambio inevitable que nos transforma a todos. Lo que se pide de él es un compromiso con la superación personal, con el aprendizaje, con los alumnos, con la creación de una sociedad mejor y con la revolución educativa y social que se requiere urgentemente (...). (Pereda, s. f., p. 4)

El docente y la docente deben comprometerse, mediante la reflexión constante sobre su tarea docente, en espacios de construcción individual y grupal, y con aportes significativos, de forma tal que pueda enfrentarse con los numerosos dilemas y retos que se presentan en este milenio.

El docente universitario, en esta nueva educación, desempeñará nuevas funciones, tales como: 
- Acompañar, orientar y guiar el trabajo y la búsqueda del estudiante.

- Promover el desarrollo integral y el mejoramiento continuo del estudiante.

- Apoyar y sostener el esfuerzo irrenunciable del estudiante.

- Diseñar escenarios, procesos y experiencias de aprendizaje significativo y relevante.

- Preparar a los estudiantes para que se adapten a la cultura vigente y, especialmente, prepararlos para el futuro.

Puesta la mirada en los perfiles estudiantiles, en las nuevas tareas que debe realizar el docente universitario, en la concepción de la formación sobre la base de competencias y basada en los autores Escudero (s. f.), Galvis (2007), Pereda (s. f.), Prieto (2005) y Ortega (2010), se delinean los siguientes perfiles para el docente universitario.

\section{Conocimiento amplio en lo disciplinar y pedagógico}

a. Conocimiento disciplinario: Propias del docente en una determinada asignatura o área disciplinar (Ortega, 2010). Actualmente, “(...) al profesor universitario se le exige y reconoce el dominio de los conocimientos de sus respectivas áreas de conocimiento, y también la competencia demostrada (proyectos, publicaciones, etc.) en lo que concierne a su papel de creador y constructor del saber, su divulgación y proyección sobre unas u otras formas de desarrollo científico y tecnológico (...)" (Escudero, s. f., 1.1 La formación en la cultura y tradición universitaria, J 3). Por otra parte, el conocimiento disciplinar no debería estar sesgado, circunscrito a áreas de especialización, sino integrado a la multidisciplinariedad y transdisciplinariedad.

\section{b. Conocimiento pedagógico:}

(...) Implica conocer y comprender las distintas formas en que un estudiante aprende, [teorías del aprendizaje, conocer modalidades y métodos de enseñanza] y los sistemas de evaluación adecuados para dar respuestas a los nuevos retos mediante la adecuada toma de decisiones relativas a la optimización formativa (...). (Ortega, 2010, p. 318)

Sin embargo,

(...) para la mayoría del profesorado, aunque siempre hay excepciones, la formación científica e investigadora constituye una suerte de imperativo moral generalmente bien asumido e internalizado, sea por razones éticas, o también, por aspiraciones legítimas de promoción. Sobre este terreno, con toda seguridad, es sobre el que recae su energía, esfuerzo y dedicación (...). [Pero] las energías para extender su formación sobre otros dominios, por ejemplo el didáctico, suelen ser más bien escasas, y quizás marginales. (Escudero, s. f., 1.1 La formación en la cultura y tradición universitaria, I 3) 
En forma aislada, se puede observar que en los últimos años ha crecido notablemente el interés de docentes e instituciones en la formación pedagógica del profesorado. Se han abierto posgrados y se han otorgado subsidios para la realización de especialidades que están permitiendo un perfeccionamiento de los mismos. Sin embargo, la formación del profesorado debería ser una de las áreas de atención preferente en las universidades, para lo cual se tendrían que establecer políticas favorecedoras de la creación de espacios permanentes para impulsar la formación de los mismos.

Con amplios conocimientos (disciplinares y pedagógicos) el docente y la docente pueden dominar y estructurar los saberes para facilitar experiencias de aprendizaje significativo. Por lo tanto, el docente universitario debe preocuparse por su formación permanente, tanto en lo disciplinar como en lo pedagógico. Al respecto, (Escudero, s. f.) expresa que la formación profesional debe entenderse :

(...) como un fenómeno integral y sostenido en el tiempo, como una actividad individual y colectiva, con vocación de relacionar la teoría y la práctica, y, finalmente, vertebrado en torno a la resolución de problemas y la mejora de la enseñanza y el aprendizaje de los alumnos. (2.3 Los procesos y oportunidade para la formación permanente, I 3)

La formación del profesorado debe ser a lo largo de toda su carrera docente y no como un episodio aislado. Debe considerar no sólo distintas técnicas o métodos de enseñanza, sino que, fundamentalmente, centrarse en aspectos sociales, culturales y relacionar teoría y práctica, en un espacio de diálogo y experiencias significativas.

De esta manera, se podría pensar en el docente como "un filósofo, amigo de la sabiduría y del conocimiento, buscador intelectual que adecue las teorías y modelos a una realidad concreta" (Pereda, s. f., p. 4).

Se considera, en este artículo, entonces, que quien ejerce la docencia universitaria debe tener la capacidad de aprender a lo largo de la vida.

\section{Habilidades de gestión}

(...) Vinculadas a la gestión, organización y planificación eficiente de la enseñanza y de sus recursos en diferentes contextos. Este tipo de competencias resultan esenciales para que el docente pueda participar en la planificación y desarrollo de objetivos, características, metodología y recursos disponibles. (Ortega, 2010, p. 319)

\section{Función de tutor o tutora}

Orienta en el auto aprendizaje del estudiantado, creando un ambiente propicio para el aprendizaje individual y colectivo. Incentiva al alumno a descubrir los diversos motivos que lo animen para ser constante, persistente y responsable en sus estudios y trabajos (Sánchez, 2001). "Dirige, (...) da sentido y fortalece el esfuerzo de sus alumnos, conduciéndolos a una sociedad con mayor libertad, con múltiples alternativas pero también cargada de incertidumbre en la cual como diría Heráclito: lo único permanente es el cambio" (Pereda, s. f., p. 4). 


\section{Capacidades culturales y contextuales}

Resulta imprescindible poseer una cultura general, conocer al estudiantado y los productos culturales con los se relaciona. Esto permitirá superar el discurso identitario ${ }^{2}$ que se dirige, de manera constante y terrible, a la juventud, y así lograr un aprendizaje desde el contexto.

\section{Capacidades comunicativas}

Vinculadas a la capacidad discursiva, o sea, a la posibilidad de apropiarse de diferentes recursos del lenguaje a nivel verbal y no verbal, los cuales le permitan transmitir al docente experiencias y provocar aprendizajes. Favorece la comunicación docente-estudiante y permite establecer un diálogo con los contextos. Se trata, pues, de una comunicación cuidadosamente elaborada, sin descuidos, rigurosa, clara, bien estructurada y, especialmente, dirigida al interlocutor.

\section{Capacidades sociales}

Relacionadas con acciones de relación social y colaboración con otras personas, el trabajo en equipo y el liderazgo para favorecer el interaprendizaje entre docentes y entre docentes y estudiantes.

\section{Capacidades metacognitivas}

(...) Relacionadas con la capacidad crítica, autocrítica y reflexiva del docente con el objetivo de que éste sea capaz de revisar su actuación docente y mejorarla de forma sistemática, así como la capacidad de reacción ante situaciones conflictivas, novedosas o imprevistas, la creatividad y la innovación didáctica y la toma de decisiones mediante la previa identificación del problema, recopilación de toda la información y propuesta de soluciones. (Ortega, 2010, p. 318)

\section{Capacidades tecnológicas}

Relacionadas con el aprendizaje, la investigación y el uso de las posibilidades que las tecnologías de la información y la comunicación brindan a la labor profesional docente. Pone énfasis en una reflexión profunda que le posibilite al docente realizar una mediación pedagógica de las mismas y, de esa forma, en los procesos de búsqueda, selección y síntesis de la información, establecer puentes entre estas y el estudiantado.

Se ha denominado discurso identitario a una mirada incapaz de ver al otro desde su cultura y sus razones, experiencias y sentires. Un discurso que coloca todo del lado de quien mira, de quien juzga, de quien atribuye ciertos valores, saberes, conductas, y niega los demás (Prieto, 2005). 


\section{Características de investigación}

Estas les permitan a los docentes y a las docentes:

a. Construir “(...) proyectos futuros integrales que ubiquen y motiven el quehacer de los alumnos en este mundo, con una concepción de lo que es el ser humano, sus posibilidades y trascendencia" (Pereda, s. f., p. 4).

b. Buscar nuevas metodologías, información y recursos para su propia formación y la del alumnado.

c. Enseñar a pensar, a descubrir, a formular y a buscar.

Además de las características mencionadas que debería tener el docente universitario, se considera que, por sobre todo, debe poseer ciertas cualidades que lo caractericen como un ser humano capaz de emprender la difícil y gratificante tarea de la docencia, entre las que destacan: honestidad, principios éticos y sensibilidad con el otro.

Los perfiles especificados anteriormente no deben considerarse en forma separada, más bien se debería:

(...) pensar en el perfil integral del profesor universitario que puede concebirse como el conjunto organizado y coherente de atributos o características altamente deseables en un educador, que se materializan en los conocimientos que posee, las destrezas que muestra, las actitudes que asume y los valores que enriquecen su vida personal y educativa. La sinergia de este conjunto de atributos le permitirá desempeñarse eficientemente, con sentido creador y crítico, en las funciones de docencia, investigación/creación, extensión y servicio que corresponden a su condición académica, concebidas como funciones interdependientes, comprometidas en el logro de la misión de la universidad. (Segura, 2004, pp. 19-20)

Para concluir y a modo de síntesis, el docente debe ser "(...) un maestro de la vida... que ponga en el centro de su vocación los valores humanos, solo así esta tendrá sentido y podrá recobrar el lugar social que le corresponde al lado de los transformadores y forjadores de la sociedad" (Pereda, s. f., p. 5).

\section{Conclusión}

La educación basada en competencias se perfila como la tendencia en materia de innovación de los modelos universitarios. Requiere que el sistema educativo, en su totalidad, se enfrente al cambio, por lo que la universidad debe proveer los mecanismos necesarios para su aplicación con respecto a políticas, herramientas, sistemas de apoyo al docente, infraestructura, relaciones institucionales, entre otros.

En cuanto a la actuación docente se requiere de procesos continuos de reflexión e instancias formales de capacitación del profesorado. Esto permitirá conducir la innovación y no adaptarse a ella, lo que revalorizará el quehacer diario en las aulas.

Sin embargo, cualquier innovación será en vano sin el compromiso docente. Esta formación necesita ineludiblemente de la responsabilidad y madurez del mismo, no sólo para , sino también para transferir su aprendizaje y experiencias en la práctica docente. 
En este ensayo se han esbozado algunos perfiles docentes como una primera aproximación, ya que los propios docentes deben reflexionar sobre su acción como educadores y, a partir de allí, retroalimentar y plantear acciones para su mejora. Son el docente y la docente universitarios quienes deben participar en la elaboración del modelo docente, proceso en el cual se debe respetar la individualidad, la especialización y la experiencia de cada uno. Esta difícil tarea se requiere enfrentar, por los docentes, con una legítima aspiración de cambiar este nuevo paradigma educativo: meta que, con compromiso y madurez, se podrá lograr.

\section{Referencias bibliográficas}

Declaración de Bolonia. (1999). The Bologna Declaration on the European space for higher education: an explanation [La Declaración de Bolonia en el espacio europeo de educación superior: una explicación]. Recuperado de http://ec.europa.eu/education/policies/educ/ bologna/bologna.pdf

Escudero, J. M. (s. f.). La formación permanente del profesorado universitario: cultura, contenidos y procesos. Portal de Desarrollo Académico [Ponencia]. Recuperado de http://www.umayor. cl/dda/Tutorial\%20Calidad\%20de\%20la\%20Docencia/page4.html

Galvis, R. V. (2007, enero-diciembre). De un perfil docente tradicional a un perfil docente basado en competencias. Acción pedagógica, 16, 48-57. Recuperado de http://www.saber.ula.ve/ bitstream/123456789/17284/2/articulo5.pdf

López, A. y Farfán. P. E. (2005, junio). El enfoque por competencias en la educación [Mesa 3, Ponencia 6]. Ponencia presentada en el Quinto Congreso Nacional y Cuarto Internacional "Retos y expectativas de la universidad" México., Universidad Autónoma de Tamaulipas. Tampico, Tamaulipas Recuperado de http://www.congresoretosyexpectativas.udg.mx/ Framesetmemorias.htm

Martell, F. (2010, enero-marzo). La competencia comunicativa, elemento central de la docencia. Revista Caminos abiertos, 179. Recuperado de http://caminosabiertos2010.blogspot. com/2010/01/la-competencia-comunicativa-elemento.html

Ortega, M. (2010). Competencias emergentes del docente ante las demandas del espacio europeo de educación superior. Revista Española de Educación Comparada 16, 305-327. Recuperado de http://www.uned.es/reec/pdfs/16-2010/14 ortega.pdf

Pereda, M. (s. f.). Perfil del docente en el siglo XXI, en el marco de un modelo educativo basado en competencias. MOPDF.com Recuperado de www.anfeca.unam.mx/.../29\%20PERFIL\%20 DEL\%20DOCENTE\%20EN\%20EL\%20SIGLO\%20XX

Prieto, D. (2005). La enseñanza en la universidad. Mendoza, Argentina: Ed. Centro Universitario.

Proyecto Tuning América Latina. (2005). “Tuning” Afinar las estructuras educativas de Europa. (Proyecto piloto apoyado por la Comisión Europea en el marco del programa Sócrates). Recuperado de http://www.tuning.unideusto.org/tuningal/ 
Rodríguez, M. C., Aguilar, A., Jiménez, M. y Pérez, S. (2001). Educación basada en competencias. ¿Necesario el conocimiento en el personal de salud? Rev. Enferm IMSS, 9(3), 133-136. Recuperado de www.imss.gob.mx/NR/rdonlyres/EC8FB70E.../3 133136.pdf

Sánchez, F. (2001, febrero). Las tutorías y la construcción de ambientes de aprendizaje en la educación abierta y a distancia. Acción Educativa, 1(1), 1-13. Recuperado de http://www. ciberdocencia.gob.pelindex.php?id=1176\&a=articulo completo

Segura, M. (2004, enero-junio). Hacia un perfil del docente universitario. Revista Ciencias de la Educación. Año 4, 1(23), 9-28.

Tobón, S. (2006). Aspectos básicos de la formación basada en competencias. Talca: Proyecto Mesesup. Recuperado de http://www.uv.mx/facpsi/proyectoaula/documents/Lectura5.pdf

Zenteno, M. (2009, noviembre). La actitud del docente frente al cambio. Retos a los que se enfrenta. Trabajo presentado en el XXII Congreso Nacional de Enseñanza de las Matemáticas. 